\title{
SOBOLEV SPACES WITH WEIGHTS IN DOMAINS AND BOUNDARY VALUE PROBLEMS FOR DEGENERATE ELLIPTIC EQUATIONS*
}

\author{
S. V. LOTOTSKY ${ }^{\dagger}$
}

\begin{abstract}
A family of Banach spaces is introduced to control the interior smoothness and boundary behavior of functions in a general domain. Interpolation, embedding, and other properties of the spaces are studied. As an application, a certain degenerate second-order elliptic partial differential equation is considered.
\end{abstract}

1. Introduction. Let $G$ be a domain in $\mathbb{R}^{d}$ with a non-empty boundary $\partial G$ and $\rho_{G}(x)=\operatorname{dist}(x, \partial G)$. For $1 \leq p<\infty$ and $\theta \in \mathbb{R}$ define the space $L_{p, \theta}(G)$ as follows:

$$
L_{p, \theta}(G)=\left\{u: \int_{G}|u(x)|^{p} \rho_{G}^{\theta-d}(x) d x<\infty\right\} .
$$

Then we can define the spaces $H_{p, \theta}^{m}(G), m=1,2, \ldots$, so that

$$
H_{p, \theta}^{m}(G)=\left\{u: u, \rho_{G} D u, \ldots, \rho_{G}^{m} D^{m} u \in L_{p, \theta}\right\}
$$

where $D^{k}$ denotes generalized derivative of order $k$. The objective of the current paper is to define spaces $H_{p, \theta}^{\gamma}(G), \gamma \in \mathbb{R}$, so that, for positive integer $\gamma$, the spaces $H_{p, \theta}^{\gamma}(G)$ coincide with the ones introduced above. It will be shown that these spaces can be easily defined using the spaces $H_{p}^{\gamma}\left(\mathbb{R}^{d}\right)$ of Bessel potentials. Note that $u \in$ $H_{p, d-p}^{1}(G)$ if and only if $u / \rho_{G}, D u \in L_{p}(G)$, which means that, for bounded $G$, the space $H_{p, d-p}^{1}(G)$ coincides with the space $\stackrel{\circ}{H}_{p}^{1}(G)$. As a result, the spaces $H_{p, \theta}^{\gamma}(G)$ can be considered as a certain generalization of the usual Sobolev spaces on $G$ with zero boundary conditions. A major application of the spaces $H_{p, \theta}^{\gamma}(G)$ is in the analysis of the Dirichlet problem for stochastic parabolic equations $[5,7]$.

Some of the spaces $H_{p, \theta}^{\gamma}(G)$ have been studied before. Lions and Magenes [6] introduced what corresponds to $H_{2, d}^{\gamma}(G)$. They constructed the scale by interpolating between the positive integer $\gamma$ for $\gamma>0$ and used duality for $\gamma<0$. Krylov [3] defined the spaces $H_{p, \theta}^{\gamma}\left(\mathbb{R}_{+}^{d}\right)$, where $\mathbb{R}_{+}^{d}$ is the half-space. After that, if $G$ is sufficiently regular and bounded, then $H_{p, \theta}^{\gamma}(G)$ can be defined using the partition of unity, and this was done in [7]. Other related examples and references can be found in Chapter 3 of [10].

In this paper, an intrinsic definition (not involving $\mathbb{R}_{+}^{d}$ ) of the spaces $H_{p, \theta}^{\gamma}(G)$ is given for a general domain $G$, and the basic properties of the spaces are studied. Once a suitable definition of the spaces is found, most of the properties follow easily from the known results. Definition and properties of the spaces $H_{p, \theta}^{\gamma}(G)$ are presented in Sections 2, 3, and 4. Roughly speaking, the index $\gamma$ controls the smoothness inside the domain, and the index $\theta$ controls the boundary behavior. In particular, the space $H_{p, \theta}^{\gamma}(G)$ with sufficiently large $\gamma$ and $\theta<0$ contains functions that are continuous in the closure of $G$ and vanish on the boundary. In Section 5 some results are presented about solvability of certain degenerate elliptic equations in a general domain $G$.

\footnotetext{
${ }^{*}$ Received Sept 7, 1999.

†Department of Mathematics, M. I. T., Cambridge, MA 02139-4307, USA (lototsky@math. mit.edu). This work was partially supported by the NSF grant DMS-9972016.
} 
Throughout the paper, $D^{m}$ denotes a partial derivative of order $m$, that is, $D^{m}=$ $\partial^{m} / \partial x_{1}^{m_{1}} \cdots \partial x_{d}^{m_{d}}$ for some $m_{1}+\cdots+m_{d}=m$. For two Banach spaces, $X, Y$, notation $X \subset Y$ means that $X$ is continuously embedded into $Y$.

2. Definition and main properties of the weighted spaces in domains. Let $G \subset \mathbb{R}^{d}$ be a domain (open connected set) with non-empty boundary $\partial G$, and $c>1$, a real number. Denote by $\rho_{G}(x), x \in G$, the distance from $x$ to $\partial G$. For $n \in \mathbb{Z}$ and a fixed integer $k_{0}>0$ define the subsets $G_{n}$ of $G$ by

$$
G_{n}=\left\{x \in G: c^{-n-k_{0}}<\rho_{G}(x)<c^{-n+k_{0}}\right\} .
$$

Let $\left\{\zeta_{n}, n \in \mathbb{Z}\right\}$ be a collection of non-negative functions with the following properties:

$$
\zeta_{n} \in C_{0}^{\infty}\left(G_{n}\right),\left|D^{m} \zeta_{n}(x)\right| \leq N(m) c^{m n}, \sum_{n \in \mathbb{Z}} \zeta_{n}(x)=1
$$

The function $\zeta_{n}(x)$ can be constructed by mollifying the characteristic (indicator) function of $G_{n}$. If $G_{n}$ is an empty set, then the corresponding $\zeta_{n}$ is identical zero.

If $u \in \mathcal{D}^{\prime}(G)$, that is, $u$ is a distribution on $C_{0}^{\infty}(G)$, then $\zeta_{n} u$ is extended by zero to $\mathbb{R}^{d}$ so that $\zeta_{n} u \in \mathcal{D}^{\prime}\left(\mathbb{R}^{d}\right)$. The space $H_{p, \theta}^{\gamma}(G)$ is defined as a collection of those $u \in \mathcal{D}^{\prime}(G)$, for which $\zeta_{n} u$ is in $H_{p}^{\gamma}$ and the norms $\left\|\zeta_{n} u\right\|_{H_{p}^{\gamma}}, n \in \mathbb{Z}$, behave in a certain way. Recall [10, Section 2.3.3] that the space of Bessel potentials $H_{p}^{\gamma}$ is the closure of $C_{0}^{\infty}\left(\mathbb{R}^{d}\right)$ in the norm $\left\|\mathcal{F}^{-1}\left(1+|\xi|^{2}\right)^{\gamma / 2} \mathcal{F} \cdot\right\|_{L_{p}\left(\mathbb{R}^{d}\right)}$, where $\mathcal{F}$ is the Fourier transform with inverse $\mathcal{F}^{-1}$.

DeFINITION 2.1. Let $G$ be a domain in $\mathbb{R}^{d}, \theta$ and $\gamma$, real numbers, and $p \in$ $(1,+\infty)$. Take a collection $\left\{\zeta_{k}, n \in \mathbb{Z}\right\}$ as above. Then

$$
H_{p, \theta}^{\gamma}(G):=\left\{u \in \mathcal{D}^{\prime}(G):\|u\|_{H_{p, \theta}^{\gamma}(G)}^{p}:=\sum_{n \in \mathbb{Z}} c^{n \theta}\left\|\zeta_{-n}\left(c^{n} \cdot\right) u\left(c^{n} \cdot\right)\right\|_{H_{p}^{\gamma}}^{p}<\infty\right\} .
$$

Since $H_{p}^{\gamma_{1}} \subset H_{p}^{\gamma_{2}}$ for $\gamma_{1}>\gamma_{2}$, the definition implies that $H_{p, \theta}^{\gamma_{1}}(G) \subset H_{p, \theta}^{\gamma_{2}}(G)$ for $\gamma_{1}>\gamma_{2}$ and all $\theta \in \mathbb{R}, 1 \leq p<\infty$. Still, it is necessary to establish correctness of Definition 2.1 by showing that the norms defined according to (2.1) are equivalent for every admissible choice of the numbers $c, k_{0}$ and the functions $\zeta_{n}$. Proving this equivalence is the main goal of this section.

Proposition 2.2. 1. If $u$ is compactly supported in $G$, then $u \in H_{p, \theta}^{\gamma}(G)$ if and only if $u \in H_{p}^{\gamma}$.

2. The set $C_{0}^{\infty}(G)$ is dense in every $H_{p, \theta}^{\gamma}(G)$.

3. If $\gamma=m$ is a non-negative integer, then

$$
H_{p, \theta}^{\gamma}(G)=\left\{u: \rho_{G}^{k} D^{k} u \in L_{p, \theta}(G), 0 \leq k \leq m\right\},
$$

where $L_{p, \theta}(G)=L_{p}\left(G, \rho_{G}^{\theta-d}(x) d x\right)$.

4. If $\left\{\xi_{n}, n \in \mathbb{Z}\right\}$ is a system of function so that $\xi_{n} \in C_{0}^{\infty}\left(G_{n}\right),\left|D^{m} \xi_{n}(x)\right| \leq$ $N(m) c^{m n}$, then

$$
\sum_{n \in \mathbb{Z}} c^{n \theta}\left\|\xi_{-n}\left(c^{n} \cdot\right) u\left(c^{n} \cdot\right)\right\|_{H_{p}^{\gamma}}^{p} \leq N\|u\|_{H_{p, \theta}^{\gamma}}^{p}
$$


with $N$ independent of $u$, and if in addition $\sum_{n} \xi(x) \geq \delta>0$ for all $x \in G$, then the reverse inequality also holds.

Proof. 1. The result is obvious because, for compactly supported $u$, the sum in (2.1) contains only finitely many non-zero terms.

2. Given $u \in H_{p, \theta}^{\gamma}(G)$, first approximate $u$ by $u_{K}=u \cdot \sum_{|k| \leq K} \zeta_{k}$, and then mollify $u_{K}$.

3. The result follows because, for all $\nu \in \mathbb{R}$ and all $x$ in the support of $\zeta_{-n}$, $N_{1} \leq c^{-\nu n} \rho_{G}^{\nu}(x) \leq N_{2}$ with $N_{1}$ and $N_{2}$ independent of $n, \nu, x$.

4. Use that, by Theorem 4.2.2 in [9], $C_{0}^{\infty}\left(\mathbb{R}^{d}\right)$ functions are pointwise multipliers in every $H_{p}^{\gamma}$.

REMARK 2.3. In the future we will also use a system of non-negative $C_{0}^{\infty}\left(\mathbb{R}^{d}\right)$ functions $\left\{\eta_{n}, n \in \mathbb{Z}\right\}$ with the following properties: $\eta_{n}$ is supported in $\left\{x: c^{-n-k_{0}-1}\right.$ $\left.<\rho_{G}(x)<c^{-n+k_{0}+1}\right\}, \eta(x)=1$ on the support of $\zeta_{n},\left|D^{m} \eta_{n}(x)\right| \leq N(m) c^{m n}$. By Proposition 2.2(4) the functions $\eta_{n}$ can replace $\zeta_{n}$ in (2.1).

Proposition 2.4. 1. For every $p \in(1, \infty)$ and $\theta, \gamma \in \mathbb{R}$, the space $H_{p, \theta}^{\gamma}(G)$ is a reflexive Banach space with the dual $H_{p^{\prime}, \theta^{\prime}}^{-\gamma}(G)$, where $1 / p+1 / p^{\prime}=1$ and $\theta / p+\theta^{\prime} / p^{\prime}=$ $d$.

2. If $0<\nu<1, \gamma=(1-\nu) \gamma_{0}+\nu \gamma_{1}, 1 / p=(1-\nu) / p_{0}+\nu / p_{1}$, and $\theta=$ $(1-\nu) \theta_{0}+\nu \theta_{1}$, then

$$
H_{p, \theta}^{\gamma}(G)=\left[H_{p_{0}, \theta_{0}}^{\gamma_{0}}(G), H_{p_{1}, \theta_{1}}^{\gamma_{1}}(G)\right]_{\nu}
$$

where $[X, Y]_{\nu}$ is the complex interpolation space of $X$ and $Y$ (see [10, Section 1.9] for the definition and properties of the complex interpolation spaces).

Proof. Let $l_{p}^{\theta}\left(H_{p}^{\gamma}\right)$ be the set of sequences with elements from $H_{p}^{\gamma}$ and the norm

$$
\left\|\left\{f_{n}\right\}\right\|_{l_{p}^{\theta}\left(H_{p}^{\gamma}\right)}^{p}=\sum_{n \in \mathbb{Z}} c^{n \theta}\left\|f_{n}\right\|_{H_{p}^{\gamma}}^{p}
$$

Define bounded linear operators $S_{p, \theta}: H_{p, \theta}^{\gamma}(G) \rightarrow l_{p}^{\theta}\left(H_{p}^{\gamma}\right)$ and $R_{p, \theta}: l_{p}^{\theta}\left(H_{p}^{\gamma}\right) \rightarrow$ $H_{p, \theta}^{\gamma}(G)$ as follows:

$$
\left(S_{p, \theta} u\right)_{n}(x)=\zeta_{-n}\left(c^{n} x\right) u\left(c^{n} x\right), \quad R_{p, \theta}\left(\left\{f_{n}\right\}\right)(x)=\sum_{n \in \mathbb{Z}} \eta_{-n}(x) f_{n}\left(c^{-n} x\right)
$$

Note that $R_{p, \theta} S_{p, \theta}=\operatorname{Id}_{H_{p, \theta}^{\gamma}(G)}$. Then, by Theorem 1.2.4 in [10], the space $H_{p, \theta}^{\gamma}(G)$ is isomorphic to $S_{p, \theta}\left(H_{p, \theta}^{\gamma}(G)\right)$, which is a closed subspace of a reflexive Banach space $l_{p}^{\theta}\left(H_{p}^{\gamma}\right)$. This means that $H_{p, \theta}^{\gamma}(G)$ is also a reflexive Banach space. The interpolation result (2.3) follows from Theorems 1.2.4 and 1.18.1 in [10].

Denote by $(\cdot, \cdot)$ the duality between $H_{p}^{\gamma}$ and $H_{p^{\prime}}^{-\gamma}$. If $v \in H_{p^{\prime}, \theta^{\prime}}^{-\gamma}(G)$, then, by the Hölder inequality, $v$ defines a bounded linear functional on $H_{p, \theta}^{\gamma}(G)$ as follows:

$$
u \mapsto\langle v, u\rangle=\sum_{n} c^{n d}\left(v_{n}, u_{n}\right)
$$

where $u_{n}(x)=\zeta_{-n}\left(c^{n} x\right) u\left(c^{n} x\right)$ and $v_{n}(x)=\eta_{-n}\left(c^{n} x\right) v\left(c^{n} x\right)$. Note that if $u, v \in$ $C_{0}^{\infty}(G)$, then $\langle v, u\rangle=\int_{G} u(x) v(x) d x$. 
Conversely, if $V$ is a bounded linear functional on $H_{p, \theta}^{\gamma}(G)$, then we use the HahnBanach theorem and the equality $\left(l_{p}^{\theta}\left(H_{p}^{\gamma}\right)\right)^{\prime}=l_{p^{\prime}}^{-\theta p^{\prime} / p}\left(H_{p^{\prime}}^{-\gamma}\right)$ to construct $v \in H_{p^{\prime}, \theta^{\prime}}^{-\gamma}(G)$ so that $V(u)=\langle v, u\rangle$.

One consequence of (2.3) is the interpolation inequality

$$
\|u\|_{H_{p, \theta}^{\gamma}(G)}=\epsilon\|u\|_{H_{p, \theta_{0}}^{\gamma_{0}}(G)}+N(\nu, p, \epsilon)\|u\|_{H_{p, \theta_{1}}^{\gamma_{1}}(G)}, \epsilon>0 .
$$

Corollary 2.5. The space $H_{p, \theta}^{\gamma}$ does not depend, up to equivalent norms, on the specific choice of the numbers $c$ and $k_{0}$ and the functions $\zeta_{n}$. Moreover, the distance function $\rho_{G}$ can be replaced with any measurable function $\rho$ satisfying $N_{1} \rho_{G}(x) \leq$ $\rho(x) \leq N_{2} \rho_{G}(x)$ for all $x \in G$, with $N_{1}, N_{2}$ independent of $x$.

Proof. By Proposition 2.2(3), we have the result for non-negative integer $\gamma$. For general $\gamma>0$ the result then follows from (2.3), where we take $p_{0}=p_{1}=p$, $\theta_{0}=\theta_{1}=\theta$, and integer $\gamma_{0}, \gamma_{1}$. After that, the result for $\gamma<0$ follows by duality.

In view of Corollary 2.5, it will be assumed from now on that $c=2$ and $k_{0}=1$.

REMARK 2.6. If $X$ is a Banach space of generalized functions on $\mathbb{R}^{d}$, then we can define the space $X_{\theta}(G)$ according to $(2.1)$ by replacing the norm $\|\cdot\|_{H_{p}^{\gamma}}$ with $\|\cdot\|_{X}$. In particular, we can define the spaces $B_{p, q ; \theta}^{\gamma}(G)$ and $F_{p, q ; \theta}^{\gamma}(G)$ using the spaces $B_{p, q}^{\gamma}$ and $F_{p, q}^{\gamma}$ described in Section 2.3.1 of [10]. Results similar to Propositions 2.2 and 2.4 can then be proved in the same way.

ExAmple. (cf. [5, Definition 1.1].) Let $G=\mathbb{R}_{+}^{d}=\left\{x=\left(x_{1}, \ldots, x_{d}\right) \in \mathbb{R}^{d}: x_{1}>\right.$ $0\}$ and $\zeta \in C_{0}^{\infty}\left(\left(b_{1}, b_{2}\right)\right), 0<b_{1}, b_{2}>3 b_{1}$. Define $\zeta(x)=\zeta\left(x_{1}\right)$ and

$$
H_{p, \theta}^{\gamma}=\left\{u \in \mathcal{D}^{\prime}(G):\|u\|_{H_{p, \theta}^{\gamma}}^{p}:=\sum_{n \in \mathbb{Z}} e^{n \theta}\left\|\zeta u\left(e^{n} \cdot\right)\right\|_{H_{p}^{\gamma}}^{p}<\infty\right\} .
$$

It follows that $H_{p, \theta}^{\gamma}=H_{p, \theta}^{\gamma}\left(\mathbb{R}_{+}^{d}\right)$ with $H_{p, \theta}^{\gamma}\left(\mathbb{R}_{+}^{d}\right)$ defined according to (2.1), where $c=e, \rho_{G}(x)=x_{1}, \zeta_{n}(x)=\zeta\left(e^{n} x\right) / \sum_{k} \zeta\left(e^{k} x\right)$, and $k_{0}$ is the smallest positive integer for which $b_{1}>e^{-k_{0}}, b_{2}<e^{k_{0}}$.

3. Pointwise multipliers, change of variables, and localization. A function $a=a(x)$ is a pointwise multiplier in a liner normed function space $X$ if the operation of multiplication by $a$ is defined and continuous in $X$. To describe the pointwise multipliers in the space $H_{p, \theta}^{\gamma}(G)$, we need some preliminary constructions. For $\gamma \in \mathbb{R}$ define $\gamma^{\prime} \in[0,1)$ as follows. If $\gamma$ is an integer, then $\gamma^{\prime}=0$; if $\gamma$ is not an integer, then $\gamma^{\prime}$ is any number from the interval $(0,1)$ so that $|\gamma|+\gamma^{\prime}$ is not an integer. The space of pointwise multipliers in $H_{p}^{\gamma}$ is given by

$$
B^{|\gamma|+\gamma^{\prime}}= \begin{cases}L_{\infty}\left(\mathbb{R}^{d}\right), & \gamma=0 \\ C^{n-1,1}\left(\mathbb{R}^{d}\right), & |\gamma|=n=1,2, \ldots \\ C^{|\gamma|+\gamma^{\prime}}\left(\mathbb{R}^{d}\right), & \text { otherwise }\end{cases}
$$

where $C^{n-1,1}\left(\mathbb{R}^{d}\right)$ is the set of functions from $C^{n-1}\left(\mathbb{R}^{d}\right)$ whose derivatives of order $n-1$ are uniformly Lipschitz continuous. In other words, if $u \in H_{p}^{\gamma}$ and $a \in B^{|\gamma|+\gamma^{\prime}}$, then

$$
\|a u\|_{H_{p}^{\gamma}} \leq N(\gamma, d, p)\|a\|_{B|\gamma|+\gamma^{\prime}}\|u\|_{H_{p}^{\gamma}} .
$$

For non-negative integer $\gamma$ this follows by direct computation, for positive non-integer $\gamma$, from Corollary 4.2.2(ii) in [9], and for negative $\gamma$, by duality.

For $\nu \geq 0$, define the space $A^{\nu}(G)$ as follows: 
1. if $\nu=0$, then $A^{\nu}(G)=L_{\infty}(G)$;

2. if $\nu=m=1,2, \ldots$, then

$$
\begin{gathered}
A^{\nu}(G)=\left\{a: a, \rho_{G} D a, \ldots, \rho_{G}^{m-1} D^{m-1} a \in L_{\infty}(G), \rho_{G}^{m} D^{m-1} a \in C^{0,1}(G)\right\} \\
\|a\|_{A^{\nu}(G)}=\sum_{k=0}^{m-1}\left\|\rho_{G}^{k} D^{k} a\right\|_{L_{\infty}(G)}+\left\|\rho_{G}^{m} D^{m} a\right\|_{C^{0,1}(G)}
\end{gathered}
$$

3. if $\nu=m+\delta$, where $m=0,1,2, \ldots, \delta \in(0,1)$, then

$$
\begin{gathered}
A^{\nu}(G)=\left\{a: a, \rho_{G} D a, \ldots, \rho_{G}^{m} D^{m} a \in L_{\infty}(G), \rho_{G}^{\nu} D^{m} a \in C^{\delta}(G)\right\}, \\
\|a\|_{A^{\nu}(G)}=\sum_{k=0}^{m}\left\|\rho_{G}^{k} D^{m} a\right\|_{L_{\infty}(G)}+\left\|\rho_{G}^{\nu} D^{m} a\right\|_{C^{\delta}(G)} .
\end{gathered}
$$

Note that, for every $a \in A^{\nu}(G)$ and $n \in \mathbb{Z}$,

$$
\left\|\zeta_{-n}\left(2^{n} \cdot\right) a\left(2^{n} \cdot\right)\right\|_{B^{\nu}} \leq N\|a\|_{A^{\nu}(G)}
$$

with $N$ independent of $n$.

THEOREM 3.1. If $a \in A^{|\gamma|+\gamma^{\prime}}(G)$, then

$$
\|a u\|_{H_{p, \theta}^{\gamma}(G)} \leq N(d, \gamma, p)\|a\|_{A|\gamma|+\gamma^{\prime}(G)} \cdot\|u\|_{H_{p, \theta}^{\gamma}(G)} .
$$

Proof. We have to show that $\left\|\eta_{-n}\left(2^{n} \cdot\right) a\left(2^{n} \cdot\right)\right\|_{B|\gamma|+\gamma^{\prime}} \leq N\|a\|_{A|\gamma|+\gamma^{\prime}(G)}$ with constant $N$ independent of $n$. The result is obvious for $\gamma=0$; for $|\gamma| \in(0,1]$ it follows from the inequality (with $\delta=|\gamma|+\gamma^{\prime}$ )

$$
\begin{aligned}
\left|\eta_{-n}(x) a(x)-\eta_{-n}(y) a(y)\right| \leq \eta_{-n}(x) \rho_{G}^{-\delta}(x)\left|a(x) \rho_{G}^{\delta}(x)-a(y) \rho_{G}^{\delta}(y)\right| \\
\quad+|a(y)|\left|\eta_{-n}(x)-\eta_{-n}(y)\right|+\eta_{-n}(x) \rho_{G}^{-\delta}(x)|a(y)|\left|\rho_{G}^{\delta}(x)-\rho_{G}^{\delta}(y)\right|
\end{aligned}
$$

and the observation that both $2^{n} \eta_{-n}$ and $\rho_{G}$ are uniformly Lipschitz continuous. If $|\gamma|>1$, we apply the same arguments to the corresponding derivatives.

Next, we study the following question: for what mappings $\psi: G_{1} \rightarrow G_{2}$ is the operator $u(\cdot) \mapsto u(\psi(\cdot))$ continuous from $H_{p, \theta}^{\gamma}\left(G_{2}\right)$ to $H_{p, \theta}^{\gamma}\left(G_{1}\right)$ ?

THEOREM 3.2. Suppose that $G_{1}$ and $G_{2}$ are domains with non-empty boundaries and $\psi: G_{1} \rightarrow G_{2}$ is a $C^{1}$-diffeomorphism so that $\psi\left(\partial G_{1}\right)=\partial G_{2}$. For a positive integer $m$ define $\nu=\max (m-1,0)$. If $D \psi \in A^{\nu}\left(G_{1}\right)$, then, for every $\gamma \in[-\nu, m]$ and $u \in H_{p, \theta}^{\gamma}\left(G_{2}\right)$,

$$
\|u(\psi(\cdot))\|_{H_{p, \theta}^{\gamma}\left(G_{1}\right)} \leq N\|u\|_{H_{p, \theta}^{\gamma}\left(G_{2}\right)}
$$

with $N$ independent of $u$.

Proof. Denote by $\phi$ the inverse of $\psi$. If $\gamma=0$, then

$$
\|u(\psi(\cdot))\|_{H_{p, \theta}^{\gamma}\left(G_{1}\right)}^{p}=\int_{G_{2}}|u(y)|^{p} \rho_{G_{1}}^{\theta-d}(\phi(y))|D \phi(y)| d y
$$


and the result follows because uniform Lipschitz continuity of $\rho_{G_{i}}, \psi$, and $\phi$ implies that the ratio $\rho_{G_{1}}(\phi(x)) / \rho_{G_{2}}(x)$ is uniformly bounded from above and below. If $\gamma=m$, the computation is similar. After that, for $\gamma \in(0, m)$, the result follows by interpolation, and for $\gamma \in[-\nu, 0)$, by duality.

The last result in this section is about localization. It answers the following question: for what collections of $C^{\infty}(G)$ functions $\left\{\xi_{k}, k=1,2, \ldots\right\}$ are the values of $\|u\|_{H_{p, \theta}^{\gamma}(G)}^{p}$ and $\sum_{n}\left\|u \zeta_{n}\right\|_{H_{p, \theta}^{\gamma}(G)}^{p}$ comparable? To begin with, let us recall the corresponding theorem for $H_{p}^{\gamma}$.

Theorem 3.3. ([4, Lemma 6.7].) If $\left\{\xi_{k}, k=0,1, \ldots,\right\}$ is a collection of $C^{\infty}\left(\mathbb{R}^{d}\right)$ functions so that $\sup _{x} \sum_{k}\left|D^{m} \xi_{k}(x)\right| \leq M(m), m \geq 0$, then $\sum_{k \geq 0}\left\|\xi_{k} v\right\|_{H_{p}^{\gamma}}^{p} \leq N\|v\|_{H_{p}^{\gamma}}^{p}$ with $N$ independent of $v$. If in addition $\inf _{x} \sum_{k}\left|\xi_{k}(x)\right|^{p} \geq \delta$ then the reverse inequality also holds: $\|v\|_{H_{p}^{\gamma}}^{p} \leq N \sum_{k \geq 0}\left\|\xi_{k} v\right\|_{H_{p}^{\gamma}}^{p}$ with $N$ independent of $v$.

The following is the analogous result for $H_{p, \theta}^{\gamma}(G)$.

THEOREM 3.4. Suppose that $\left\{\chi_{k}, k \geq 1\right\}$ is a collection of $C^{\infty}(G)$ functions so that $\sup _{x \in G} \sum_{k} \rho_{G}^{m}(x)\left|D^{m} \chi_{k}(x)\right| \leq \bar{N}(m), m \geq 0$. Then $\sum_{k}\left\|u \chi_{k}\right\|_{H_{p, \theta}^{\gamma}(G)}^{p} \leq$ $N\|u\|_{H_{p, \theta}^{\gamma}(G)}^{p}$. If, in addition, $\inf _{x \in G} \sum_{k}\left|\chi_{k}(x)\right|^{p} \geq \delta$ for some $\delta>0$, then $\|u\|_{H_{p, \theta}^{\gamma}(G)}^{p}$ $\leq N \sum_{k}\left\|u \chi_{k}\right\|_{H_{p, \theta}^{\gamma}(G)}^{p}$.

Proof. With $\hat{\chi}_{0, n}=1-\eta_{n}, \hat{\chi}_{k, n}(x)=\chi_{k}(x) \eta_{-n}(x), k \geq 1$, we find

$$
\sum_{k \geq 1}\left\|u \chi_{k}\right\|_{H_{p, \theta}^{\gamma}(G)}^{p}=\sum_{n \in \mathbb{Z}} \sum_{k \geq 0} 2^{n \theta}\left\|\hat{\chi}_{k, n}\left(2^{n} \cdot\right) \zeta_{-n}\left(2^{n} \cdot\right) u\left(2^{n} \cdot\right)\right\|_{H_{p}^{\gamma}}^{p} .
$$

Both statements of the theorem now follow from Theorem 3.3.

EXAMPLE. (cf. [7, Section 2].) Let $G$ be a bounded domain of class $C^{|\gamma|+2}$ with a partition of unity $\chi_{0} \in C_{0}^{\infty}(G), \chi_{1}, \ldots \chi_{K} \in C_{0}^{\infty}\left(\mathbb{R}^{d}\right)$ and the corresponding diffeomorphism $\psi_{1}, \ldots, \psi_{K}$ that stretch the boundary inside the support of $\chi_{1}, \ldots, \chi_{K}$ (see, for example, Chapter 6 of [2] for details). Then an equivalent norm in $H_{p, \theta}^{\gamma}(G)$ is given by

$$
\|u\|_{H_{p, \theta}^{\gamma}(G)}=\left\|u \chi_{0}\right\|_{H_{p}^{\gamma}}+\sum_{m=1}^{K}\left\|u\left(\psi_{m}^{-1}(\cdot)\right) \chi_{m}\left(\psi_{m}^{-1}(\cdot)\right)\right\|_{H_{p, \theta}^{\gamma}\left(\mathbb{R}_{+}^{d}\right)} .
$$

Indeed, writing $\sim$ to denote the equivalent norms, we deduce from Proposition 2.2(1) and Theorems 3.2 and 3.4 that

$$
\|u\|_{H_{p, \theta}^{\gamma}(G)} \sim \sum_{m=0}^{K}\left\|u \chi_{m}\right\|_{H_{p, \theta}^{\gamma}(G)} \sim\left\|u \chi_{0}\right\|_{H_{p}^{\gamma}}+\sum_{m=1}^{K}\left\|u\left(\psi_{m}^{-1}(\cdot)\right) \chi_{m}\left(\psi_{m}^{-1}(\cdot)\right)\right\|_{H_{p, \theta}^{\gamma}\left(\mathbb{R}_{+}^{d}\right)} .
$$

4. Further properties of the spaces $H_{p, \theta}^{\gamma}(G)$. Let $\rho=\rho(x)$ be a $C^{\infty}(G)$ function so that $N_{1} \rho_{G}(x) \leq \rho(x) \leq N_{2} \rho_{G}(x)$ and $\left|\rho_{G}^{m}(x) D^{m+1} \rho(x)\right| \leq N(m)$ for all $x \in G$ and for every $m=0,1, \ldots$ In particular, $\rho(x)=0$ on $\partial G$ and all the first-order partial derivatives of $\rho$ are pointwise multipliers in every $H_{p, \theta}^{\gamma}(G)$. An example of the function $\rho$ is

$$
\rho(x)=\sum_{n \in \mathbb{Z}} 2^{-n} \zeta_{n}(x)
$$


where the functions $\zeta_{n}$ are as in Section 2 with $c=2$.

THEOREM 4.1. 1. The following conditions are equivalent:

- $u \in H_{p, \theta}^{\gamma}(G)$;

- $u \in H_{p, \theta}^{\gamma-1}(G)$ and $\rho D u \in H_{p, \theta}^{\gamma-1}(G)$;

- $u \in H_{p, \theta}^{\gamma-1}(G)$ and $D(\rho u) \in H_{p, \theta}^{\gamma-1}(G)$.

In addition, under either of these conditions, the norm $\|u\|_{H_{p, \theta}^{\gamma}(G)}$ can be replaced by $\|u\|_{H_{p, \theta}^{\gamma-1}(G)}+\|\rho D u\|_{H_{p, \theta}^{\gamma-1}(G)}$ or by $\|u\|_{H_{p, \theta}^{\gamma-1}(G)}+\|D(\rho u)\|_{H_{p, \theta}^{\gamma-1}(G)}$.

2. For every $\nu, \gamma \in \mathbb{R}$,

(4.1) $\rho^{\nu} H_{p, \theta}^{\gamma}(G)=H_{p, \theta-p \nu}^{\gamma}(G)$ and $\|\cdot\|_{H_{p, \theta-p \nu}^{\gamma}(G)}$ is equivalent to $\left\|\rho^{-\nu} \cdot\right\|_{H_{p, \theta}^{\gamma}(G)}$.

Proof. It is sufficient to repeat the arguments from the proofs of, respectively, Theorem 3.1 and Corollary 2.6 in [3].

Corollary 4.2. 1. If $u \in H_{p, \theta}^{\gamma}(G)$, then

$$
D u \in H_{p, \theta+p}^{\gamma-1}(G) \text { and }\|D u\|_{H_{p, \theta+p}^{\gamma-1}(G)} \leq N(d, \gamma, p, \theta)\|u\|_{H_{p, \theta}^{\gamma}}(G) .
$$

2. If $\rho_{G}$ is a bounded function (for example, if $G$ is a bounded domain), then $H_{p, \theta_{1}}^{\gamma}(G) \subset H_{p, \theta_{2}}^{\gamma}(G)$ for $\theta_{1}<\theta_{2}$ and $H_{p}^{\gamma}(G) \subset H_{p, \theta}^{\gamma}(G)$ for $\theta \geq d$.

Recall the following notations for continuous functions $u$ in $G$ :

$$
\|u\|_{C(G)}=\sup _{x \in G}|u(x)|,[u]_{C^{\nu}(G)}=\sup _{x, y \in G} \frac{|u(x)-u(y)|}{|x-y|^{\nu}}, \nu \in(0,1) .
$$

THEOREM 4.3. Assume that $\gamma-d / p=k+\nu$ for some $k=0,1, \ldots$ and $\nu \in(0,1)$. If $u \in H_{p, \theta}^{\gamma}(G)$, then

$$
\sum_{k=0}^{m}\left\|\rho^{k+\theta / p} D^{k} u\right\|_{C(G)}+\left[\rho^{m+\nu+\theta / p} D^{m} u\right]_{C^{\nu}(G)} \leq N(d, \gamma, p, \theta)\|u\|_{H_{p, \theta}^{\gamma}(G)}
$$

Proof. It is sufficient to repeat the arguments from the proof of Theorem 4.1 in [3]. प

Note that if $u \in H_{p, \theta}^{\gamma}(G)$ with $\gamma>1+d / p$ and $\theta<0$, then, by Theorem 4.3,u is continuously differentiable in $G$ and is equal to zero on the boundary of $G$. This is one reason why the spaces $H_{p, \theta}^{\gamma}(G)$ can be considered as a generalization of the usual Sobolev spaces with zero boundary conditions.

5. Degenerate elliptic equations in general domains. Throughout this section, $G \subset \mathbb{R}^{d}$ is a domain with a non-empty boundary but otherwise arbitrary, and $\rho$ is the function introduced at the beginning of Section 4. Consider a second-order elliptic differential operator

$$
\mathcal{L}=a^{i j}(x) D_{i} D_{j}+\frac{b^{i}(x)}{\rho(x)} D_{i}-\frac{c(x)}{\rho^{2}(x)},
$$

where $D_{i}=\partial / \partial x_{i}$ and summation over the repeated indices is assumed. A related but somewhat different operator is studied in Section 6 of [10]. The objective of this 
section is to study solvability in $H_{p, \theta}^{\gamma}(G)$ of the equation $\mathcal{L} u=f$. It follows from Theorem 4.3 that, for appropriate $\theta$ and $\gamma$, the solution of the equation will also be a classical solution of the Dirichlet problem $\mathcal{L} u=f,\left.u\right|_{\partial G}=0$. The values of $\gamma \in \mathbb{R}, 1<p<\infty$, and $\theta \in \mathbb{R}$ will be fixed throughout the section.

The following assumptions are made.

Assumption 5.1. Uniform ellipticity: there exist $\kappa_{1}, \kappa_{2}>0$ so that, for all $x \in G$ and $\xi \in \mathbb{R}^{d}, \kappa_{1}|\xi|^{2} \leq a^{i j}(x) \xi_{i} \xi_{j} \leq \kappa_{2}|\xi|^{2}$.

Assumption 5.2. Regularity of the coefficients:

$$
\|a\|_{A^{\nu_{1}}(G)}+\|b\|_{A^{\nu_{2}(G)}}+\|c\|_{A^{|\gamma+1|+\gamma^{\prime}(G)}} \leq \kappa_{2}
$$

where $\nu_{1}=\max \left(2,|\gamma-1|+\gamma^{\prime}\right), \nu_{2}=\max \left(1,|\gamma|+\gamma^{\prime}\right)$. (See beginning of Section 3 for the definition of $\gamma^{\prime}$.)

Note that under assumption 5.2 the operator $\mathcal{L}$ is bounded from $H_{p, \theta-p}^{\gamma+1}(G)$ to $H_{p, \theta+p}^{\gamma-1}(G)$. Therefore, we say that $u \in H_{p, \theta-p}^{\gamma+1}(G)$ is a solution of $\mathcal{L} u=f$ with $f \in H_{p, \theta+p}^{\gamma-1}(G)$ if the equality $\mathcal{L} u=f$ holds in $H_{p, \theta+p}^{\gamma-1}(G)$.

THEOREM 5.1. Under Assumptions 5.1 and 5.2, there exists a $c_{0}>0$ depending only on $d, p, \theta$, the function $\rho$, and the coefficients $a, b$ so that, for every $f \in H_{p, \theta+p}^{\gamma-1}(G)$ and every $c(x)$ satisfying $c(x) \geq c_{0}$, the equation $\mathcal{L} u=f$ has a unique solution $u \in H_{p, \theta-p}^{\gamma+1}(G)$ and $\|u\|_{H_{p, \theta-p}^{\gamma+1}(G)} \leq N\|f\|_{H_{p, \theta+p}^{\gamma-1}(G)}$ with the constant $N$ depending only on $d, \gamma, p, \theta$, the function $\rho$, and the coefficients $a, b, c$.

To prove Theorem 5.1, we first establish the necessary a priori estimates, then prove the theorem for some special operator $\mathcal{L}$, and finally use the method of continuity to extend the result to more general operators.

Lemma 5.2. If $u \in H_{p, \theta-p}^{\gamma+1}(G)$ and Assumptions 5.1 and 5.2 hold, then

$$
\|u\|_{H_{p, \theta-p}^{\gamma+1}(G)} \leq N\left(\|\mathcal{L} u\|_{H_{p, \theta+p}^{\gamma-1}(G)}+\|u\|_{H_{p, \theta-p}^{\gamma-1}(G)}\right)
$$

with $N$ independent of $u$.

Proof. Assume first that $b=c=0$. Define $u_{n}(x)=\zeta_{-n}\left(2^{n} x\right) u\left(2^{n} x\right)$ and the operator

$$
\mathcal{A}_{n}=\left(a^{i j}\left(2^{n} x\right) \eta_{-n}\left(2^{n} x\right)+\left(1-\eta_{-n}\left(2^{n} x\right) \delta^{i j}\right)\right) D_{i j}
$$

where $\eta$ is as in Remark 2.3. Clearly, $\left\|u_{n}\right\|_{H_{p}^{\gamma+1}} \leq N\left(\left\|\mathcal{A} u_{n}\right\|_{H_{p}^{\gamma-1}}+\|u\|_{H_{p}^{\gamma-1}}\right)$, and, by (3.1), $N$ is independent of $n$. On the other hand,

$$
\mathcal{A}_{n} u_{n}(x)=2^{2 n}\left(\zeta_{-n} \mathcal{L} u+2 a^{i j} D_{i} \zeta_{-n} D_{j} u+a^{i j} u D_{i j} \zeta_{-n}\right)\left(2^{n} x\right)
$$

It remains to use the inequalities $\|D u\|_{H_{p}^{\gamma-1}} \leq N\|u\|_{H_{p}^{\gamma}} \leq \epsilon\|u\|_{H_{p}^{\gamma+1}}+N \epsilon^{-1}\|u\|_{H_{p}^{\gamma-1}}$ with sufficiently small $\epsilon$, and then sum up the corresponding terms according to (2.1).

If $b, c$ are not zero, then

$$
\left\|a^{i j} D^{i j} u\right\|_{H_{p, \theta+p}^{\gamma-1}(G)} \leq\|\mathcal{L} u\|_{H_{p, \theta+p}^{\gamma-1}(G)}+N\|u\|_{H_{p, \theta-p}^{\gamma}(G)}+N\|u\|_{H_{p, \theta-p}^{\gamma-1}(G)}
$$

and the result follows from the interpolation inequality (2.4). 
LEMMA 5.3. If Assumptions 5.1 and 5.2 hold, then there exists a $c_{0}>0$ depending on $d, p, \theta$, the function $\rho$, and the coefficients $a, b$, so that, for every $c(x)$ satisfying $c(x) \geq c_{0}$ and every $u \in L_{p, \theta}(G)$,

$$
\|u\|_{L_{p, \theta}(G)} \leq N\left\|\rho^{2} \mathcal{L} u\right\|_{L_{p, \theta}(G)}
$$

with $N$ independent of $u$.

Proof. It is enough to consider $u \in C_{0}^{\infty}(G)$. Writing $f=-\rho^{2} \mathcal{L} u$, we multiply both sides by $|u|^{p-2} u \rho^{\theta-d}$ and integrate by parts similar to the proof of Theorem 3.16 in [3]. The result is

$$
\int_{G} f|u|^{p-2} u \rho^{\theta-d} d x=\int_{G}(c(x)+h(x))|u|^{p} \rho^{\theta-d} d x,
$$

where $|h(x)| \leq N_{h}$ and $N_{h}$ depends on $d, p, \theta$, and $\|a\|_{A^{2}(G)}+\|b\|_{A^{1}(G)}+\|D \rho\|_{A^{1}(G)}$. It remains to take $c_{0}=2 N_{h}$ and use the Hölder inequality.

It follows from Lemmas 5.2 and 5.3 that if $c(x) \geq c_{0}$ and $\gamma \geq 1$, then

$$
\|u\|_{H_{p, \theta-p}^{\gamma+1}(G)} \leq N\|\mathcal{L} u\|_{H_{p, \theta+p}^{\gamma-1}(G)} .
$$

Lemma 5.4. There exists a $\bar{c}>0$ depending on $p, \theta, \gamma$, and the function $\rho$ so that the operator $\rho^{2}(x) \Delta-\bar{c}$ is a homeomorphism from $H_{p, \theta}^{\gamma+1}(G)$ to $H_{p, \theta}^{\gamma-1}(G)$.

Proof. Keeping in mind that $\rho \in C^{0,1}(G)$ and $\rho(x)=0$ on $\partial G$, let $\bar{\rho}$ be a $C^{0,1}\left(\mathbb{R}^{d}\right)$ extension of $\rho$ so that $\bar{\rho} \in C^{\infty}(G-\partial G)$. Consider a family of diffusion processes $\left(X_{t}^{x}, x \in \mathbb{R}^{d}, t \geq 0\right)$ defined by

$$
X_{t}^{x}=x+\sqrt{2} \int_{0}^{t} \bar{\rho}\left(X_{s}^{x}\right) d W_{s}
$$

where $\left(W_{t}, t \geq 0\right)$ is a standard $d$-dimensional Wiener process on some probability space $(\Omega, \mathcal{F}, P)$ (see, for example, Chapter V of [1] or Chapter I of [8]). Note that, by uniqueness, $X_{t}^{x}=x$ if $x \in \partial G$, and $X_{t}^{x} \in G$ for all $t>0$ as long as $x \in G$. Theorems (3.3) and (3.9) from Chapter I of [8] imply that, with probability one, both $D X_{t}^{x}$ and its inverse are in $C(G)$ for all $t \geq 0$. Further analysis shows that, for every $p>1$ and every positive integer $m$,

$$
E\left\|D X_{t}^{x}\right\|_{A^{m}(G)}^{p}+E\left\|D\left(X_{t}^{x}\right)^{-1}\right\|_{A^{m}(G)}^{p} \leq N_{1} e^{N_{2} t}
$$

with constants $N_{1}$ and $N_{2}$ depending on $p, m$.

Assume that $f \in C_{0}^{\infty}(G)$ and define

$$
u(x)=-E \int_{0}^{\infty} f\left(X_{t}^{x}\right) e^{-\bar{c} t} d t
$$

By Theorem 5.8.5 in [1], there exists a $c_{1}>0$ depending only on $d$ and $\bar{\rho}$ so that, for $\bar{c}>c_{1}$, the function $u$ is twice continuously differentiable in $G$ and $\bar{\rho}^{2}(x) \Delta u(x)-$ $\bar{c} u(x)=f(x)$ for all $x \in G$. On the other hand, after repeating the proof of Theorem 3.2 and using (5.2), we conclude that there exists a $c_{2}$ depending on $d, \gamma, \bar{\rho}$ so that, for $\bar{c}>c_{2}$ and for every $\gamma \in \mathbb{R}$, the function $u$ belongs to $H_{p, \theta}^{\nu}(G)$ and

$$
\|u\|_{H_{p, \theta}^{\gamma}(G)} \leq N\|f\|_{H_{p, \theta}^{\gamma}(G)}
$$


The statement of Lemma 5.4 now follows.

Proof of Theorem 5.1. Take $\bar{c}$ as in Lemma 5.4 and define the operators $\mathcal{L}_{0}=$ $\Delta-\bar{c} / \rho^{2}(x)$ and $\overline{\mathcal{L}}_{0}=\rho^{2}(x) \Delta-\bar{c}$. Lemmas 5.4 and Theorem 4.1(2) imply that, for all $\gamma, \theta \in \mathbb{R}$ and $1<p<\infty$, these operators are homeomorphisms from $H_{p, \theta-p}^{\gamma+1}(G)$ to, respectively, $H_{p, \theta+p}^{\gamma-1}(G)$ and $H_{p, \theta-p}^{\gamma-1}(G)$.

Assume first that $\gamma \geq 1$. Then a priory estimate (5.1) and the method of continuity (using the operators $\left.\lambda \mathcal{L}+(1-\lambda) \mathcal{L}_{0}, 0 \leq \lambda \leq 1\right)$ imply the conclusion of the theorem.

If $\nu<1$, then assume first that $0 \leq \nu<1$. For $f \in H_{p, \theta+p}^{\nu-1}(G)$, define $u=$ $\overline{\mathcal{L}}_{0} \mathcal{L}^{-1}\left(\overline{\mathcal{L}}_{0}^{-1} f\right)-\mathcal{L}^{-1}(\bar{f})$, where $\bar{f}=\left(\mathcal{L} \overline{\mathcal{L}}_{0}-\overline{\mathcal{L}}_{0} \mathcal{L}\right) \mathcal{L}^{-1}\left(\overline{\mathcal{L}}_{0}^{-1} f\right)$. Direct computations show that

- $\bar{f} \in H_{p, \theta+p}^{\nu}(G)$ and $\|\bar{f}\|_{H_{p, \theta+p}^{\nu}(G)} \leq N\|f\|_{H_{p, \theta+p}^{\nu-1}(G)}$;

- $u$ is well defined, $u \in H_{p, \theta-p}^{\nu+1}(G),\|u\|_{H_{p, \theta-p}^{\nu+1}(G)} \leq N\|f\|_{H_{p, \theta+p}^{\nu-1}(G)}$, and $\mathcal{L} u=f$. This process can be repeated as many time as necessary. Theorem 5.1 is proved.

REMARK 5.5. It follows from Theorem 4.3 that, if the conditions of Theorem 5.1 hold with $\gamma>d / p+2$ and $\theta<p$, then the function $u$ is the classical solution of

$$
a^{i j}(x) D_{i j} u+\frac{b^{i}(x)}{\rho(x)} D_{i} u-\frac{c(x)}{\rho^{2}(x)} u=f, x \in G ;\left.\quad u\right|_{\partial G}=0 .
$$

Acknowledgment. I wish to thank Professor David Jerison and Professor Daniel Stroock for very helpful discussions.

\section{REFERENCES}

[1] N. V. KRYLOV, Introduction to the Theory of Diffusion Processes, American Mathematical Society, Providence, RI, 1995.

[2] N. V. KRYLOV, Lectures on Elliptic and Parabolic Equations in Hölder Spaces, American Mathematical Society, Graduate Studies in Mathematics 12, Providence, RI, 1996.

[3] N. V. KRYLOV, Weighted Sobolev spaces and Laplace's and the heat equations in a half space, Communications in PDE, 24:9-10 (1999), pp. 1611-1653.

[4] N. V. KRYLOV, An analytic approach to SPDEs, in Stochastic Partial Differential Equations. Six Perspectives, Mathematical Surveys and Monographs, B. L. Rozovskii and R. Carmona, ed., AMS, 1999, pp. 185-242.

[5] N. V. KRYLOV AND S. V. Lototsky, A Sobolev space theory of SPDEs with constant coefficients in a half space, SIAM J. Math. Anal., 31 (2000), pp. 19-33.

[6] J. L. Lions AND E. Magenes, Non-homogeneous Boundary Value Problems and Applications,I, Springer-Verlag, Berlin, 1972.

[7] S. V. Lототsкy, Dirichlet problem for stochastic parabolic equations in smooth domains, Stoch. Stoch. Reports, 68:1-2 (1999), pp. 145-175.

[8] D. W. STroocK, Topics in Stochastic Differential Equations, Tata Institute of Fundamental Research, Bombay, India, 1982.

[9] H. Triebel, Theory of Function Spaces II, Birkhauser, Basel, 1992.

[10] H. TRIEBEL, Interpolation Theory, Function Spaces, Differential Operators, Johann Amrosius Barth, Heidelberg, 1995. 